\title{
PROPUESTA METODOLÓGICA PARA ANALIZAR LA SEGREGACIÓN SOCIO-ESPACIAL EN EL PERIURBANO DE CIUDADES INTERMEDIAS EN MÉXICO
}

\author{
Cinthia Fabiola Ruiz López
}

Centro de Investigaciones en Geografía Ambiental, Universidad Nacional Autónoma de México cruiz@ciga.unam.mx

ORCID iD: https://orcid.org/0000-0002-2852-4338

\section{Yadira Mireya Méndez-Lemus}

Centro de Investigaciones en Geografía Ambiental, Universidad Nacional Autónoma de México ymendez@ciga.unam.mx

ORCID iD: https://orcid.org/0000-0002-9381-9722

\section{José Antonio Vieyra Medrano}

Centro de Investigaciones en Geografía Ambiental, Universidad Nacional Autónoma de México avieyra@ciga.unam.mx

ORCID iD: https://orcid.org/0000-0003-2094-1919.

ORCID iD Colectivo: https://orcid.org/0000-0003-2513-7612

Cómo citar este artículo/citation: Ruíz López, Cinthia Fabiola; Méndez-Lemus, Yadira Mireya y Vieyra Medrano, José Antonio (2021). Propuesta metodológica para analizar la segregación socio-espacial en el periurbano de ciudades intermedias en México. Estudios Geográficos, 82 (290), e060. https://doi.org/10.3989/estgeogr.202072.072

\begin{abstract}
Resumen: El crecimiento de las ciudades latinoamericanas ha sido fragmentado y sectorizado, generando condiciones desiguales para sus pobladores. En México, las disparidades en la conformación espacial de los centros urbanos han aumentado junto con la segregación, principalmente en el espacio de expansión urbano-rural de ciudades intermedias.

En general, la forma en que se ha abordado el estudio de la segregación ha sido descriptivo, centrado en variables socioeconómicas de los grupos sociales y principalmente bajo el análisis de las grandes ciudades, siendo más escasos los estudios en el periurbano; sobre todo en las ciudades intermedias. Esto hace necesario repensar los planteamientos metodológicos para analizar la segregación en dichos espacios caracterizados por su gran dinamismo y la permanencia de estructuras rurales.

Conjugando dimensiones físicas y sociales con un acercamiento multiescalar y multitemporal del territorio, este artículo propone una metodología que analiza a la segregación en el periurbano de ciudades intermedias mexicanas, poniendo en evidencia las implicaciones en la estructura urbana y la organización social de las comunidades.

Además, permite identificar a la segregación como un proceso de diferenciación social- espacial construido históricamente desde contextos regionales y municipales, ambientes que sirven de espacio para la interrelación de los pobladores, al mismo tiempo, les plantean condiciones de vida diversa, ante esas los habitantes responden reforzando o limitando su segregación. Esta propuesta contribuye a desmitificar la percepción negativa de la segregación en el periurbano de ciudades mexicanas, que bien podrían reconocerse también en ciertas ciudades latinoamericanas.
\end{abstract}

Palabras claves: Segregación socioespacial, periurbano, ciudades intermedias, prejuicios, condiciones de vida, desigualdad.

\section{METHODOLOGICAL APPROACH TO ANALYZE SOCIAL-SPATIAL SEGREGATION IN THE PERI-URBAN AREAS OF MEXICAN INTERMEDIARY CITIES}

Abstract: The growth of Latin-American cities, has been fragmented and sectorized, setting unequal conditions for the inhabitants. In Mexico, disparities in the spatial conformation of the urban centers as well as the segregation of people, have increased mainly in peri-urban areas.

The studies about segregation has been descriptive, focused mainly on socioeconomic variable of the social groups living in large cities. Only a few studies have centered the attention in peri-urban areas of the intermediary cities. It shows necessity reconsider methodological approaches to analyze segregation in peri-urban areas that have had growing urban dynamisms but keeping its rural structures.

Combine physical and social dimensions from different geographical scales and temporary, this paper proposes a methodology to analyze the segregation in peri-urban areas of Mexican intermediary cities. The methodology shows the implications of the urban structures and social organization for the communities in segregation process. 
Also, this methodology allows identifies the segregation like social differentiation process has been building historically and created in regional and municipalities contexts which are the environments where the population interrelate with different living conditions. The habitants have faced this context reinforced and limited their segregation.

This methodological approach contributes to demystify the negative perspective of segregation in the peri-urban areas of Mexican intermediary cities, which is also important for Latin American cities.

Keywords: Social-spatial segregation, peri-urban areas, intermediary cities, prejudice, living condition, inequality.

\section{INTRODUCCIÓN}

En México, los estudios sobre la segregación han sido mayormente descriptivos y centrados principalmente en las grandes urbes (Aguilar y Mateo, 2011; Aguilar, Romero, Hernández, 2015; Garrocho y Campos, 2013; 2015; Rubalcaba y Schteingart, 2012). La mayoría de estos estudios no reconocen las diferentes escalas geográficas y los cambios históricos en el proceso de segregación de un territorio. Esto hace necesario repensar la noción y entendimiento de la segregación no solo en las grandes metrópolis, sino también en ciudades medianas y pequeñas (las cuales albergan alrededor del $45 \%$ de la población urbana del país) abordando el proceso de manera compleja y multidimensional (Vaughan y Arbaci, 2011). Las ciudades intermedias son de particular interés en este estudio debido al tamaño de la población que actualmente albergan y a los procesos de crecimiento y expansión que las caracteriza.

Las ciudades intermedias se definen por su dimensión demográfica ${ }^{1}$ (cambiante en los contextos nacionales) y por la capacidad para vincular sistemas urbanos con espacios rurales o de menor jerarquía (Madrid, 2011; Pasciaroni, 2012; Roberts, 2015). En México, según la Secretaría de Desarrollo Social (SEDESOL), Consejo Nacional de Población (CONAPO) e Instituto Nacional de Estadista y Geografía (INEGI) (2010 citado en SEDESOL, ONU-HABITAT, 2011) las ciudades de tamaño mediano son de dos tipos: de 100 mil a menos de 500 mil habitantes (medias); y de $\mathbf{5 0 0}$ mil hasta 1 millón de habitantes (intermedias). Estas últimas son todas Zonas Metropolitanas y el centro de atención de este estudio debido su dinamismo (sin precedentes) de los últimos años. A pesar de que no son tan competitivas como las ciudades grandes del país, estas juegan un papel importante en la economía local y regional ya que funcionan como nodos que conectan redes nacionales e internacionales. Asimismo, la organización espacial de estas ciudades se ha adaptado a las demandas de la globalización. En este sentido, las periferias de estas urbes han sido centrales en este proceso. Por ejemplo, el establecimiento de parques industriales en dichas periferias ha creado nuevas redes y flujos de interacción con otros nodos urbanos. Por otro lado, la construcción intensiva de fracciona- mientos para la clase trabajadora ha seguido un patrón similar. Sin embargo, esta expansión del periurbano ha provocado una serie de problemas sociales y ambientales que han segregado a la población originaria y recién llegada a dichos territorios, dificultando su acceso a servicios básicos (agua, drenaje, salud y educación principalmente) y generando modificaciones en las relaciones sociales entre las localidades y dentro de ellas. En el 2018 se identificó que existían 401 ciudades. De ellas 22 son intermedias (de 500 mil a 1 millón) que albergaban al $18.5 \%$ de la población nacional según la Secretaría de Desarrollo Agrario, Territorial y Urbano (SEDATU) y CONAPO (2018).

Este artículo busca mejorar el entendimiento de la segregación en ciudades intermedias mexicanas reconociendo sus características temporales y escalares. A través de una propuesta metodológica guiada por el cuestionamiento: ¿Cómo se expresa la segregación socio-espacial en el periurbano de ciudades intermedias mexicanas?, la propuesta se basa en un enfoque fenomenológico y existencialista, de acuerdo con el cual se entiende al periurbano como un territorio construido en una relación dialéctica entre lo rural y lo urbano, formado por las acciones y resistencias de sujetos, situados espacial e históricamente (Scarlato y Costa, 2017). Desde esta visión la segregación es un proceso de diferenciación socio-espacial, socialmente construido y expresado en la distribución física e interrelación social de los habitantes en múltiples escalas territoriales (Sabatini, 2006; Thibert y Osorio, 2014; Vergara-Erices y Garín, 2016).

De acuerdo con lo anterior, se propone analizar a la segregación física y social en el periurbano de ciudades intermedias mexicanas, a través de las transformaciones en la estructura física de municipios periféricos y cambios en el estilo de vida de la población. Desde esta perspectiva se aborda a la segregación bajo tres pilares: la multidimensionalidad (física y social), la multiescalaridad espacial (localidad/fraccionamiento, barrio y familia) y la multitemporalidad, que abarca cuarenta años, observados en cortes temporales por décadas.

La relevancia de esta propuesta metodológica reside en que: 1) captura e integra las particularidades del proceso de segregación física y social de los territorios 
periurbanos de ciudades intermedias mexicanas; 2) enfatiza las múltiples escalas y temporalidades en las que se construye la segregación; y 3 ) expone un nuevo enfoque en la literatura sobre segregación en ciudades intermedias principalmente en México y aplicable para otras ciudades latinoamericanas.

El documento primero muestra el estado de la cuestión de los estudios de segregación física y social; después describir la propuesta metodológica, mostrando las etapas, escalas e instrumentos para recolectar la información en campo; y por último, se realiza una discusión de algunos resultados de la metodología en dos ciudades intermedias mexicanas.

\section{ESTADO DE LA CUESTIÓN: LA DIMENSIÓN FÍSICA Y SOCIAL DE LA SEGREGACIÓN}

El acercamiento a la segregación no es nuevo. Algunos autores lo sitúan con los estudios de la escuela de Chicago, en la década de 1920. Dos han sido las dimensiones de análisis de la segregación: la física que reflexiona sobre la concentración, homogeneidad y proximidad de grupos; y la social, explicada por las diferencias en la interrelación de los grupos sociales, influenciados por su identidad o prejuicios. ${ }^{2}$ Esta propuesta se acerca a los planteamientos de Sabatini (2006) sobre la definición compuesta de la segregación, que dan cuenta de las dimensiones diferenciables de este proceso y sus impactos sociales y espaciales.

Los estudios tradicionales de la segregación física miden el grado de homogeneidad y concentración social-espacial (Domínguez, 2017), con variables socioeconómicas del habitante, la capacidad de ahorro y el espacio donde se asienta (Aguilar et al., 2015; Garrocho y Campos, 2013; 2015; Rubalcaba y Schteingart, 2012; Sabatini, 2006; Savage, Devine, Cunningham, Taylor, Li, Hjellbrekke, Le Roux, Friedman, y Miles, 2013;); otros, clasifican a la población a partir de los estilos de vida (Aguilar y Mateo, 2011), una forma de agrupar a los pobladores desde la geodemografía que reconoce las características similares.

Desde esas visiones, la segregación se puede expresar en: igualdad, exposición, concentración, centralización y agrupamiento (Domínguez, 2017; Massey, Rothwell, Domina, 2009). Es decir, la segregación no se expresa solo con aislamiento. La perspectiva física es la más desarrollada en la literatura mexicana, pero tiene al menos cinco limitantes: no considera las dimensiones política, cultural o social de los habitantes al realizar los cálculos solo a partir del lugar de resi- dencia, desestimando la importancia de otros lugares como la calle y espacio de comunidad (Vaughan y Arbaci, 2011); los índices usados no tienen representación espacial (Garrocho y Campos, 2013); sus resultados dependen de la agrupación de los datos; no explican las interrelaciones reales al interior de las unidades de análisis; ${ }^{3}$ además, exponen solo un momento de la segregación sin incluir las diversas temporalidades (Massey et al., 2009).

Retomar esa postura para el análisis de la segregación en el periurbano permite identificar que tan dividida está la población, a partir de su distribución física, así como la superposición de diferentes expresiones de la segregación (igualdad, exposición, concentración, centralidad y agrupamiento). Pero el análisis debe superar el uso de variables socioeconómicas como única forma de distribución de la población. Por lo tanto, esta metodología considera la inclusión de rasgos poblacionales (edad, condición migratoria, población indígena, escolaridad, características de las viviendas y entorno urbano-rural) como forma de agrupar a la población a partir de características similares. Además, incluye varias temporalidades que relaciona a la segregación con el contexto vivido por sus habitantes y las modificaciones que han experimentado. Estos datos serán expresados en patrones de segregación del periurbano de ciudades intermedias, en diferentes temporalidades.

La segunda perspectiva de análisis es social, que vincula a la organización social, sistema de ideas y valores de los grupos. En ella, el centro de atención es la diferencia en las interacciones sociales, políticas, culturales y ambientales de los habitantes o grupos sociales (escolaridad, empleo, origen) y la influencia de la identidad, percepción de grupos o prejuicios en esas relaciones (Sabatini, 2006; Savage et al., 2013; Vergara-Erices y Garín, 2016). La segregación social es una dimensión y efecto de la exclusión social (Saraví, 2004), pero dista de ella al evidenciar la diferenciación en los contactos sociales e interacciones de un grupo de habitantes respecto a otros causada por rasgos socioculturales, relaciones sociales (formales e informales), prejuicios e identidades (Saraví, 2004). Mientras la exclusión social hace referencia a las prácticas sociales discriminatorias y desventajosas que limitan a los habitantes para adquirir un bien, un beneficio o servicio (Ramírez y Ziccardi, 2008). Por lo tanto, la segregación social no necesariamente implica la exclusión social. El estudio de la segregación social es menos desarrollado en la literatura mexicana. Tiene énfasis en la población étnica, etaria, relación en- 
tre los habitantes, el territorio habitado y la ciudad (Garrocho y Campos, 2015; Savage et al., 2013); su dimensión espacial es también limitada, poco se relacionan los contactos sociales con las características del espacio físico y la dimensión política (Vaughan y Arbaci, 2011); y tampoco se visualiza desde un análisis multi-temporal y escalar.

\section{PROPUESTA METODOLÓGICA}

Esta propuesta metodología pretende trascender la visión instrumental en el análisis de la segregación, que coarta la práctica y teoría a una realidad traducida por la lógica circunscrita en evidencia tangible de hechos y fenómenos positivos (Costa y Scarlato, 2019, p.642). Se retoma el enfoque fenomenológico y existencialista que permiten no reducir el análisis de la segregación a una teoría o metodología, sino considerar al espacio (periurbano) como un producto social de hechos históricos determinados y determinantes, como categoría de la totalidad dialéctica (Costa y Scarlato, 2019, p.642). Además, el enfoque fenomenológico es considerado como la representación de las interacciones causales y finales de hechos y fenómenos socio-naturales que serán observados en campo (Costa y Scarlato, 2019, p.648).

Desde los planteamientos fenomenológicos y existencialistas de Sartre (1960), Aparecida (1999), Scarlato y Costa (2017) y Costa y Scarlato (2019) se (re)define al periurbano y la segregación. Sartre ${ }^{4}$ considera que el "sujeto" primero existe luego define su esencia y toma conciencia. Para él la historia personal de un sujeto hace la historia del mundo. Para el autor, "todo lo que el hombre soporta y sus actos se reflejan en su sociedad (Sartre, 2015)" (citado en Scarlato y Costa, 2017, p.7). En ese sentido, el periurbano no define al hombre, sino éste es responsable del espacio.

Recuperando los planteamientos de Aparecida (1999) la ciudad se forma por un conjunto de redes y materialidades visibles, pero también lo abstracto que forman la totalidad (p.12). Según la autora, no se puede entender a la ciudad (o sus partes) sin considerar su complejidad y habitantes. Según Scarlato y Costa (2017) el entendimiento de la ciudad debe reconocer la historicidad de los objetos, mostrar las interrelaciones material y emocional. Para los autores la producción de la ciudad (o periurbano) es un proceso de síntesis de hechos y fenómenos, objetivos y subjetivos que se incorporan en su construcción (Scarlato y Costa, 2017, p.2). Los autores consideran que en la geografía la multiplicidad de interacciones humanas socializa la naturaleza y producen los paisajes como un momento visible y con singularidad en el espacio, lo que permite pensarlo desde la totalidad (Costa y Scarlato, 2019, p.645).

Basados en esos planteamientos, se define al periurbano como un subconcepto geográfico que sintetiza los múltiples hechos, fenómenos e interacciones sociedad -naturaleza en una relación dialéctica urbano-rural, objetivas y subjetivas, acciones y resistencias de sujetos espacial e históricamente situados. Ese entendimiento del periurbano permite definirlo desde su relación ciudad- campo, que lo acerca a la totalidad, como parte de la ciudad (múltiples escalas). Permitiendo diferenciarlo de otros conceptos utilizado para referirse al espacio que rodea a la ciudad. ${ }^{5}$ Además, esos planteamientos permiten acercarse a la segregación como un proceso de diferenciación de la población que será construido histórica y socialmente, expresado en la distribución física y las interrelaciones de habitantes en un territorio (Sabatini, 2006, p.7; Vergara-Erices y Garín, 2016, p.7).

\section{Múltiples temporalidades y escalas}

Esta propuesta metodológica pretende evidenciar las múltiples temporalidades y escalas de la segregación. Se propone como periodo de análisis de la década de 1970 al 2010. Ello porque en 1990 se da el cambio sustantivo en las tendencias de crecimiento de las ciudades intermedias como muestra otros estudios (Aguilar y Vázquez, 2000, p.93; SEDESOL, CONAPO e INEGI, 2012; SEDATU y CONAPO, 2018). El periodo de análisis será observado en cortes temporales por décadas, estableciendo la relación entre el transcurrir del tiempo y las modificaciones en el periurbano, para identificar cómo el mundo social y la acción humana se ajustan a esos cambios (Girola, 2011).

Las múltiples escalas incluyen los contextos regionales, municipales, localidades o fraccionamientos, barrios y familias, poniendo una mayor atención en las cuatro últimas. Las escalas no son continuos sino niveles imbricados, es decir lo local está subsumido a lo municipal, éste a lo regional y así sucesivamente. Las diferentes escalas permiten determinar el ritmo temporal (cotidianamente, mensualmente, excepcionalmente, etc.) como la frecuencia de las relaciones sociales en cada escala (Giménez, 1999, p.30). Aunado a ello, las diferentes escalas son útiles para identificar como la segregación se construye desde decisiones individuales y familiares, que determinan un patrón de segregación a nivel barrio, localidad, municipio y región (Van Kempen, 2007). La segregación se expresa en las inequidades y en procesos identitarios 
(Vaughan y Arbaci, 2011), que reconocen al individuo como parte de diferentes grupos sociales. Estas dos características (múltiples temporalidades y escalas) son importantes porque evidencian como se construye la segregación en los diferentes momentos históricos de acuerdo con distintos contextos (interrelaciones urbano-rurales), guiando las acciones propuestas para cada etapa de la metodología.

\section{Etapas metodológicas}

La figura 1 distingue los componentes de la propuesta (escalas $(E)$, actores $(A c)$, dimensiones $(f, s)$, interrelaciones $(h, v, t)$ y temporalidades $(T))$, organizados en 2 etapas primordiales de análisis. Se parte del supuesto que la segregación aumenta en el tiempo, debido al modelo desigual de las ciudades y las modificaciones que experimentan los habitantes del periurbano. Sus implicaciones incrementan a menor escala $(E)$ reflejadas en las desigualdades y los procesos identitarios.

Se propone, una primera etapa, reconocer los contextos: regional $(R)$ y municipal $(M)$ que plantean las condiciones externas, como el ambiente donde se interrelacionan los pobladores de las localidades. El pro- pósito es identificar cómo la segregación se construye en ambientes regionales y municipales, cambiantes en el tiempo $(T)$. La atención está en la práctica de dos actores: gobierno estatal (RAC) y municipal (MAC) respectivamente. En la dimensión física ( $R f$ y $M f$ ) se observan los cambios en las funciones del periurbano, las conexiones con la ciudad central y el tipo de población residente. En la social (Rs y Ms), centrados en aspectos políticos, dos elementos son revisados: los cambios en la imagen pública del municipio usada por el gobierno estatal y municipal para justificar la aplicación de políticas públicas; y las trasformaciones de la capacidad institucional de coordinación entre los gobiernos, a través de relaciones verticales $(v)$ y horizontales $(h)$ que establecen sus actores dentro y fuera de sus propios gobiernos. La forma cómo se organiza la sociedad a partir de las percepciones de estos actores es fundamental para comenzar a delimitar los actores clave (individuales y colectivos) para la siguiente etapa. En esta etapa se propone revisar información socioeconómica de los municipios en los Censos de Población y Vivienda (1970, 1980, 1990, 2000 y 2010) del INEGI. La imagen pública es revisada en informes de gobiernos estatales y municipales, así como entrevistas con funcionarios estales y municipales.

FIGURA 1

COMPONENTES METODOLÓGICOS PARA ANALIZAR LA SEGREGACIÓN DE ACUERDO CON LAS DISTINTAS ETAPAS DE ANÁLISIS

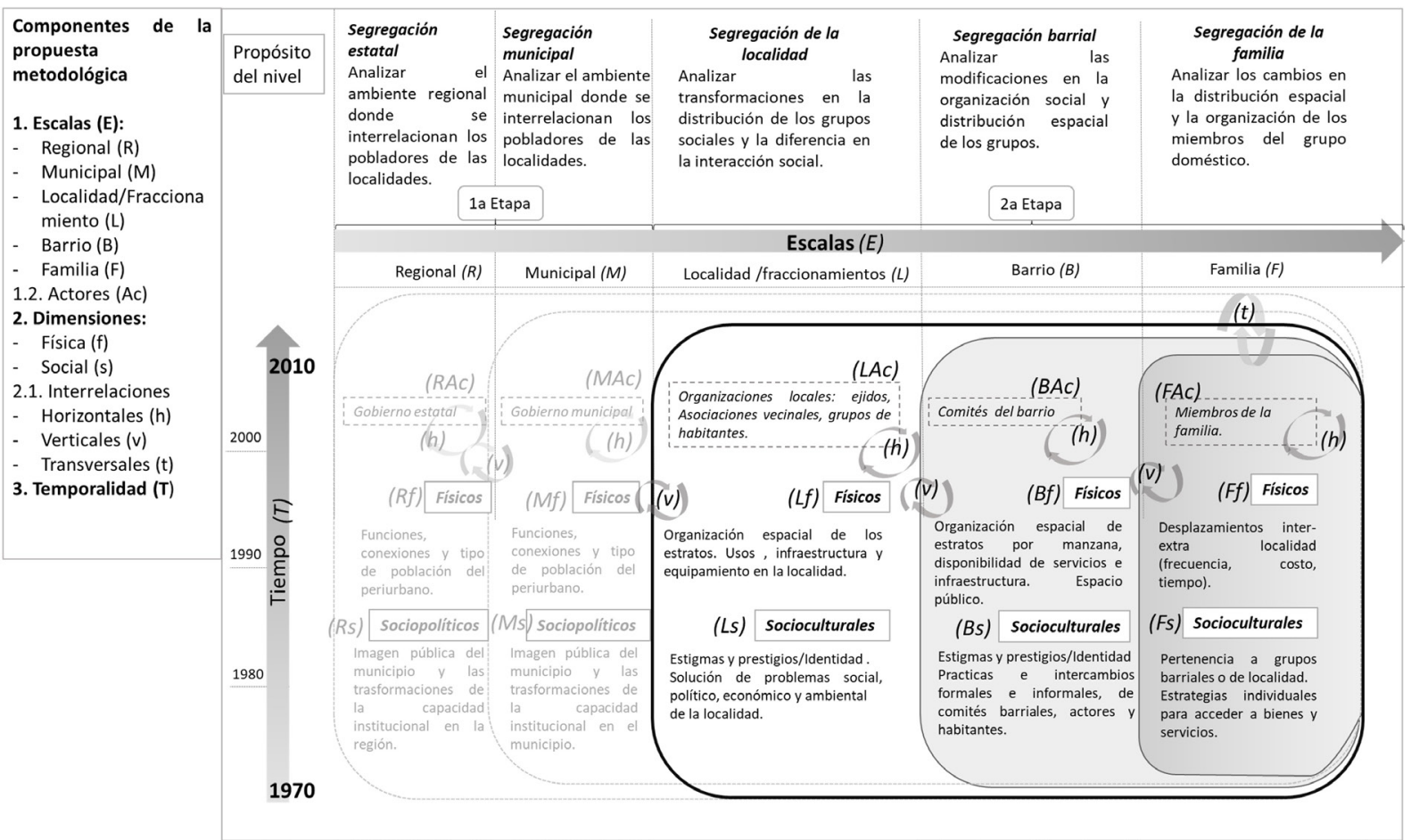

Fuente: Elaboración propia. 
En una segunda etapa, el centro de atención se enfoca en tres escalas (localidad/fraccionamiento [ $L$ ], barrio $[B]$ y familia $[F]$ ) por sus rasgos físicos y socioculturales.

\section{Localidades y fraccionamientos}

Las localidades y los fraccionamientos ( $L$ ) son espacios construidos colectivamente, con una historia en común, con normas locales que regulan la vida comunitaria, donde sus pobladores tejen relaciones por medio de las organizaciones sociales para acceder a bienes y servicios. Las localidades son: núcleos de población ejidal, comunidades agrarias (incluyen tierras de uso común, parcelas y asentamientos humanos) y fraccionamientos urbanos.

Se entenderá a la segregación en la localidad ( $L$ ), en dos dimensiones: la física ( $L f$ ) expresada en los patrones de distribución residencial, usos y condiciones de los espacios en la localidad; y la social (Ls) observada en el tipo y naturaleza de las relaciones cultivadas por los miembros de las organizaciones y su papel en la resolución de problemas municipales, coordinación con los externos, acceso a bienes y servicios, así como la mejora de la imagen pública de la localidad.

En esta escala, los actores observados son las organizaciones locales ( $L A C$ ) como: ejidos, asociación de vecinos, grupos de la tercera edad, de mujeres, jóvenes, etc. Se usa la delimitación administrativa de las localidades para identificar cómo organizan el espacio al interior de la unidad territorial. Para ello, primero se identifican los cambios en la dimensión física ( $L f)$, observados a través de cuatro observables. Primero, la distribución física de los grupos sociales en el espacio residencial. Segundo, las modificaciones de los usos del espacio público. Tercero, los cambios en la disponibilidad de servicios e infraestructura de los grupos. Cuatro, la cercanía o alejamiento a las parcelas y las áreas comunes. Se pretende definir las transformaciones en el componente físico de la localidad de ser posible, desde la década de los setenta.

En lo sociocultural (LS) se analizan los cambios en las relaciones y los valores de las organizaciones locales. En esta dimensión importan los cambios en los estratos sociales de los integrantes de las organizaciones, cambios en los espacios de acciones del grupo, en las jerarquías, en las relaciones horizontales ( $h$ ) y verticales $(v)$ con actores gubernamentales externos a la localidad. Es importante caracterizar esas relaciones en el tiempo porque permiten identificar las diferencias entre los contactos de los grupos sociales en el espacio público (Méndez-Lemus, Vieyra, GüizaValverde, Hernández-Guerrero, 2016).

En la localidad, se mide como la distribución física de los miembros de las organizaciones sociales limitan o fomentan el acceso a bienes, servicios y solución de problemas, a lo largo del tiempo $(T)$. También se analiza la diferenciación de la localidad por los estigmas, prestigios e identidad del grupo y cómo ello se ha construido, permitiendo generar ventajas y desventajas en la vida cotidiana.

La fuente de información en esta etapa son las variables socioeconómicas de los habitantes del periurbano disponible en los Censos de Población y Vivienda de 1990- $2010^{6}$ del INEGI. Complementada con información cualitativa recabada en: entrevistas a miembros de organizaciones locales y gubernamentales externos; y mapeos participativos para identificar los cambios en el acceso a bienes, servicios y solución de problemas.

\section{Barrio}

El barrio ( $B$ ) es el espacio, construido históricamente, donde el poblador realiza prácticas sociales y culturales conocidas y familiares, que le permite intercambiar bienes, información y recursos que afectan los contactos y fomentan valores y normas, generando ventajas y desventajas para sus pobladores (Saraví, 2004).

Se entiende que la segregación barrial $(B)$ es construida y determinada por los cambios en la distribución física de los grupos ( $B f$ ) y en la interacción social (Bs), ambos como una limitante o fomento en el acceso a bienes, servicios y solución de problemas barriales. En esta escala los comités del barrio (BAC) (de agua, para mejoramiento de infraestructura, de seguridad, entre otros) son el centro de atención.

En la dimensión física (Bf) de la segregación a nivel barrial se revisan los cambios en distribución espacial de los grupos sociales de diferente estrato sociodemográfico, es decir, los patrones de segregación residencial por manzana (o Áreas Geoestadísticas Básicas ${ }^{7}$ ) de $2010^{8}$. También se deben inspeccionar las modificaciones en los usos de los espacios públicos barriales (calles, plazas, parques y equipamiento) para evaluar cómo ha influido en la creación de contactos sociales, revisar los cambios en la disponibilidad de servicios, infraestructura y tipo de vivienda. Esta información es importante porque indica las formas en que los habitantes del barrio han vivido la segregación. 
En la dimensión sociocultural (Bs) hay dos aspectos esenciales que se deben considerar. Primero, las interrelaciones (horizontales, verticales y transversales) que se entretejen a este nivel y sus cambios a lo largo del tiempo. Por ejemplo, la coordinación y vinculación interna de los comités del barrio en las relaciones horizontales ( $h$ ) y con otros comités de barrios (BAC), en temas relacionados al mejoramiento del barrio, las viviendas y servicios; así como la modificación de la posición espacial de los miembros del comité. Además, en relaciones verticales $(v)$ con actores externos que se identificaron en jerarquías de análisis mayores. Segundo, en dichos comités, se identifican como han cambiado los estigmas y prestigios de los habitantes del barrio en la constitución de su identidad y la influencia de ello en el proceso de coordinación con otros comités barriales.

La fuente de información de esta etapa son las variables socioeconómicas de los habitantes del periurbano disponible en el Censo de Población y Vivienda del INEGI del 2010. La información cualitativa se recaba a través de entrevistas con miembros de los comités del barrio para identificar los estigmas, prestigio y cambios en el acceso a bienes, servicios y solución de problemas.

\section{La familia}

La escala de análisis más pequeña es la familia (F), entendida como agrupaciones de individuos con lazos de parentesco; viven bajo el mismo techo, tienen una historia en común; están organizados por un sistema de valores y normas; cuentan con un presupuesto y proyecto en común donde cada miembro cumple un papel (Gutiérrez, Díaz y Román, 2016).La segregación en la familia está determinada por desplazamientos en las prácticas cotidianas (dimensión física $[F f]$ ) y el aislamiento social (dimensión sociocultural $[F s]$ ) a lo largo de su historia de vida. Ello permite la obtención de trabajo, instrucción escolar, oferta cultural, créditos y servicios en sus viviendas.

El objetivo de incluir la escala familiar es identificar como cambia la segregación en sus miembros (FAC). Primero, en la dimensión física ( $F f$ ) se propone identificar cómo los miembros del grupo doméstico han vivido los patrones de segregación residencial (de la localidad y del barrio) y cómo sus desplazamientos cotidianos han cambiado en el tiempo $(T)$. Es importante conocer los destinos dentro de la localidad, fuera de ella, los costos, la frecuencia y el tiempo.

En la dimensión sociocultural (Fs) se caracteriza a los miembros en términos de ( $F A c$ ): edad, género, ocupación, ingreso, estrato social, religión, tipo de actividad cultural que realiza, adscripción indígena, nivel de escolaridad y antecedente migratorio.

La inclusión de la familia pretende conocer como han vivido la segregación, a partir de cinco observables. Primero, la pertenencia a grupos del barrio o de la localidad. Segundo, los estigmas y prestigios de los cuales son objeto. Tercero, los cambios en sus relaciones (verticales $[\mathrm{v}]$, horizontales $[\mathrm{h}]$ y transversales [t]). Cuarto, las estrategias familiares para acceder a trabajos, instrucción escolar, oferta cultural, créditos, vivienda y servicios de salud. Quinto, las relaciones dentro de la familia para identificar aquellos miembros que son más susceptibles de ser segregados incluso dentro del propio grupo. En este apartado, la información se recaba a través de historias de vida, que permitirá identificar la secuencia de eventos con múltiples causas e interrelaciones.

La segregación es un proceso de diferenciación socioespacial de la población, construido históricamente, de acuerdo con variables físicas y sociales, en múltiples interrelaciones en diversas escalas y temporalidades. Por ello, es relevante constatar las modificaciones de segregación física (patrones de segregación residencial) con los estigmas, prestigios e identidades de los habitantes en cada escala territorial y temporalidad. Esto generan ventajas o desventajas para un grupo social en la vida comunitaria y en el periurbano, este abordaje permite identificar cómo se refuerzan o crean nuevas formas de desigualdad urbana.

\section{APLICACIÓN DE LA METODOLOGÍA Y DISCUSIÓN DE RESULTADOS PRELIMINARES}

La metodología propuesta está actualmente siendo aplicada en dos ciudades intermedias de México: Oaxaca (al sur de la república mexicana) y Morelia (al occidente del país $)^{9}$. La elección de las urbes responde a sus tendencias de crecimiento y su ubicación en el territorio nacional, generando diferencias en el proceso de construcción social e histórica, así como de segregación (Fig. 2).

Como se mencionó, la metodología conjuga un acercamiento en múltiples dimensiones (física y social), escalas y temporalidades. En la dimensión física se utilizaron rasgos poblacionales propuestos en otros estudios (Aguilar y Matero, 2011), modificando el análisis a la luz de condiciones en la vivienda y en el entorno periurbano. En la segunda, la social, se utilizaron variables como relaciones, estigma, identidad 
FIGURA 2

UBICACIÓN DE LAS CIUDADES DE ESTUDIO (MORELIA Y OAXACA, MÉXICO)
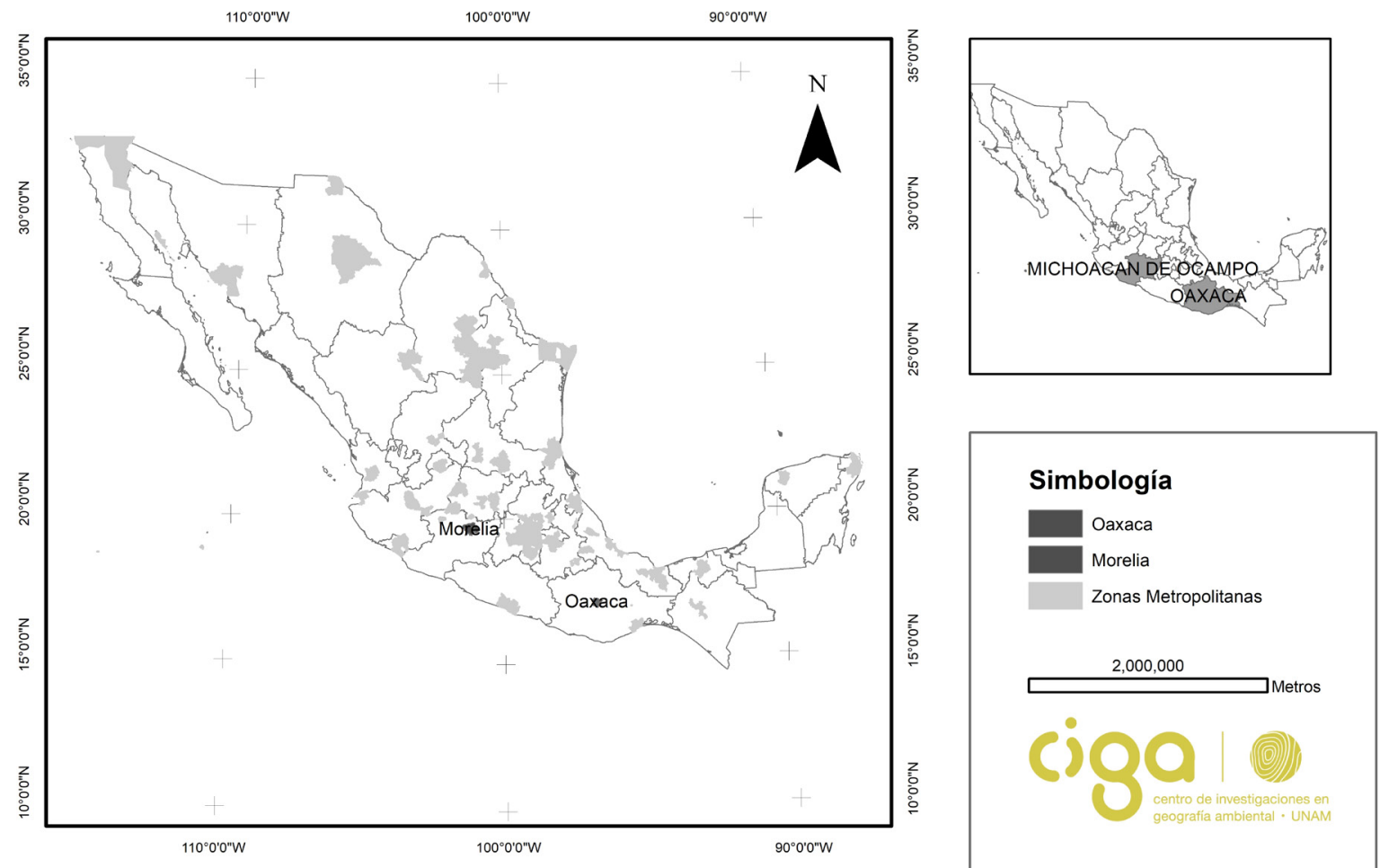

Fuente: Sistema Urbano Nacional (SEDESOL, CONAPO e INEGI, 2012). Elaboración propia

y prestigio, de acuerdo con otros estudios (Saraví, 2004), revisados en varias escalas. Conjugando las dos dimensiones se generó un acercamiento complejo a la segregación (Vaughan y Arbaci, 2011). La multiplicidad de escalas (regional, municipal, localidades, barrio y familia), visto a la luz de cambios en los últimos cuarenta años, permitió identificar la construcción social e histórica de la segregación. En los contextos regional y municipal se encontraron cambios en la distribución de funciones (económicas, habitacionales, ecológicas y culturales) de los espacios periurbanos.

La segregación física es una característica de esos cambios, la cual se concentra cercana al municipio central y es más dispersa en los municipios periféricos. Fueron tres procesos que reprodujeron la segregación en estos contextos (regional y municipal): la mixtura de funciones, la disminución de los índices de segregación y la importancia del centro. Generando una disimilitud de los municipios periféricos respecto al central, acorde a lo encontrado en otros trabajos (Thibert y Osorio, 2014, p. 1320).

En los municipios estudiados se observó una inclusión de nuevas tipologías habitacionales (fraccio- namientos, urbanizaciones cerradas, asentamientos irregulares) que se aproximan a los asentamientos tradicionales, como se observa en la figura 3. Se identificó una disminución en la segregación física al existir mayor heterogeneidad en la población (por rasgos económicos, educación, adscripción indígena, migración y edades de los habitantes). Las zonas de los municipios en los cuales fue más evidente la disminución de la segregación son las fronteras con el municipio central y las vías de comunicación. Contrario a ello, las zonas más alejadas experimentaron un proceso de dispersión de población indígena, carente de servicios salud e inactiva económicamente.

Los resultados concuerdan con la distribución dispersa de la población indígena y tipologías habitacionales encontradas por Domínguez (2017, p.179). Pero dista de lo encontrado por Álvarez del Torre (2017) sobre la centralidad en la distribución de población mayor de 64 años. Esta discrepancia encontrada en nuestros resultados es producto del uso de varias escalas en la metodología.

En la segregación social, se identificaron cambios de valoraciones del espacio periurbano y municipios 


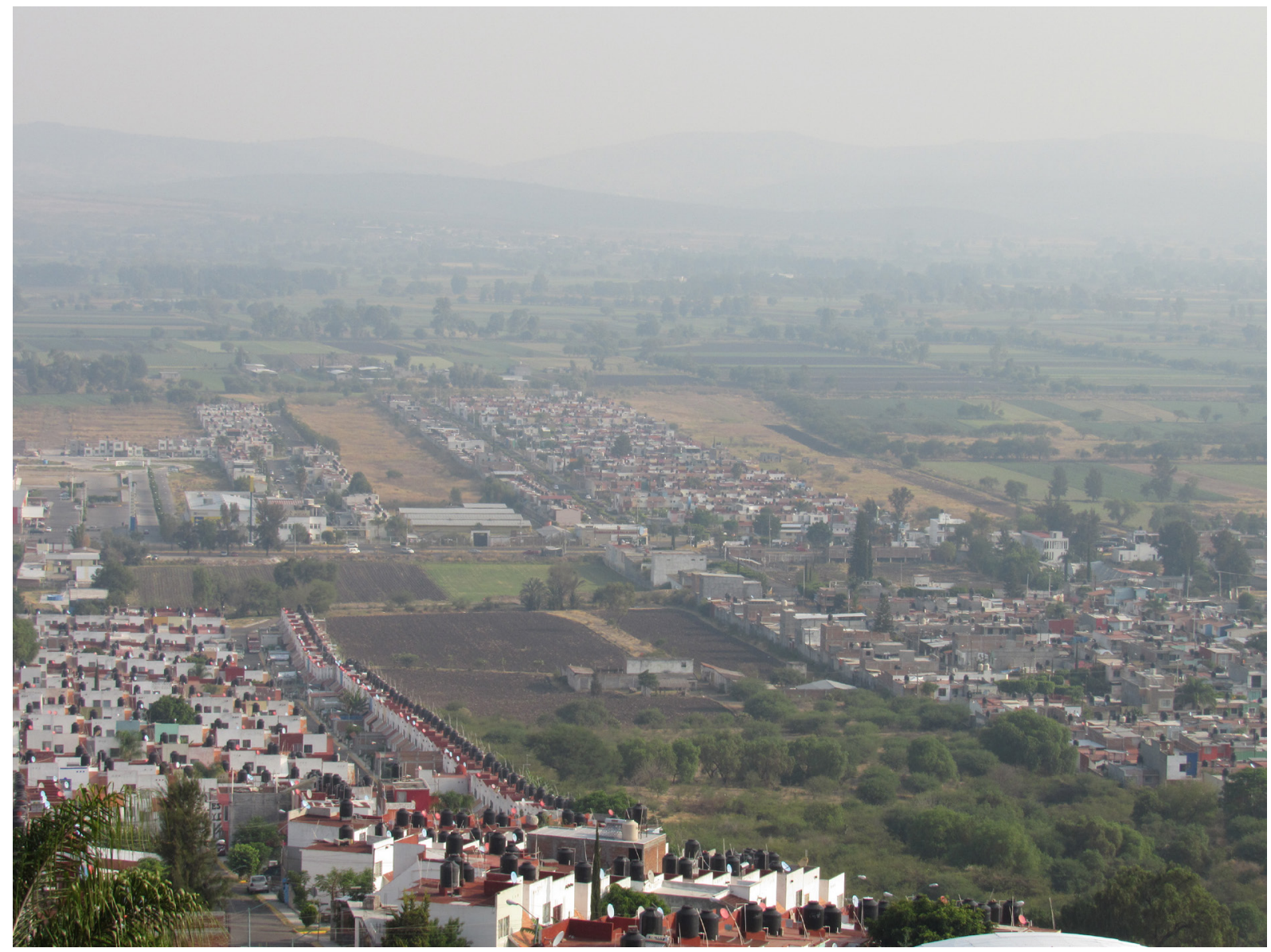

Fuente: Trabajo de campo, Morelia, México, 25 de mayo de 2019.

periféricos que ponen de manifiesto la diferenciación en procesos de planeación y gestión, así como en el acceso a servicios (educación, salud, comercio y administración pública) y fuentes de empleo para los habitantes. La disimilitud en la distribución de los recursos de los habitantes se intensificó junto al estigma, la identidad y los (des)prestigios, acorde a los planteado por Thibert y Osorio (2014, p. 1320).

En las escalas localidades y barrios, la segregación física se hace más evidente por el origen de los habitantes (asentamientos tradicionales, irregulares y fraccionamientos) más que los otros rasgos poblacionales, hallazgo que concuerdan con lo planteado por Elorza (2019, p.106) que consideran al origen de las poblaciones como un elemento diferenciador en la escala local.

En la dimensión social de esos contextos se identificaron modificaciones en las relaciones (horizontales, verticales y transversales) y valores (prestigios, estigmas e identidades) entre los actores (originales y recién llegados) que se expresan en un acceso diferencial a servicios como agua y drenaje, así como en la inclusión en la organización social (comités y asambleas). Siguiendo a Vergara-Erices y Garín (2016) ese acceso diferencial genera una exclusión funcional. Contrario a esto, se identificaron procesos organizativos locales que refuerzan las identidades locales como lo expone Elorza (2019, p.107). En la figura 4, se observa un asentamiento en proceso de consolidación que se organiza para acceder a servicios básicos (agua y drenaje).

En el caso de la escala familiar se identificó que su localización física determina sus prácticas de movilidad para el acceso a servicios (salud, educación, agua y drenaje) y oportunidades laborales. Las familias recién llegadas al periurbano son las que experimentaron mayores dificultades para acceder a los servicios fuera y dentro de las localidades. En la segregación social se observaron las valoraciones de los "otros" habitantes (originarios hacia recién llegados o viceversa) que 


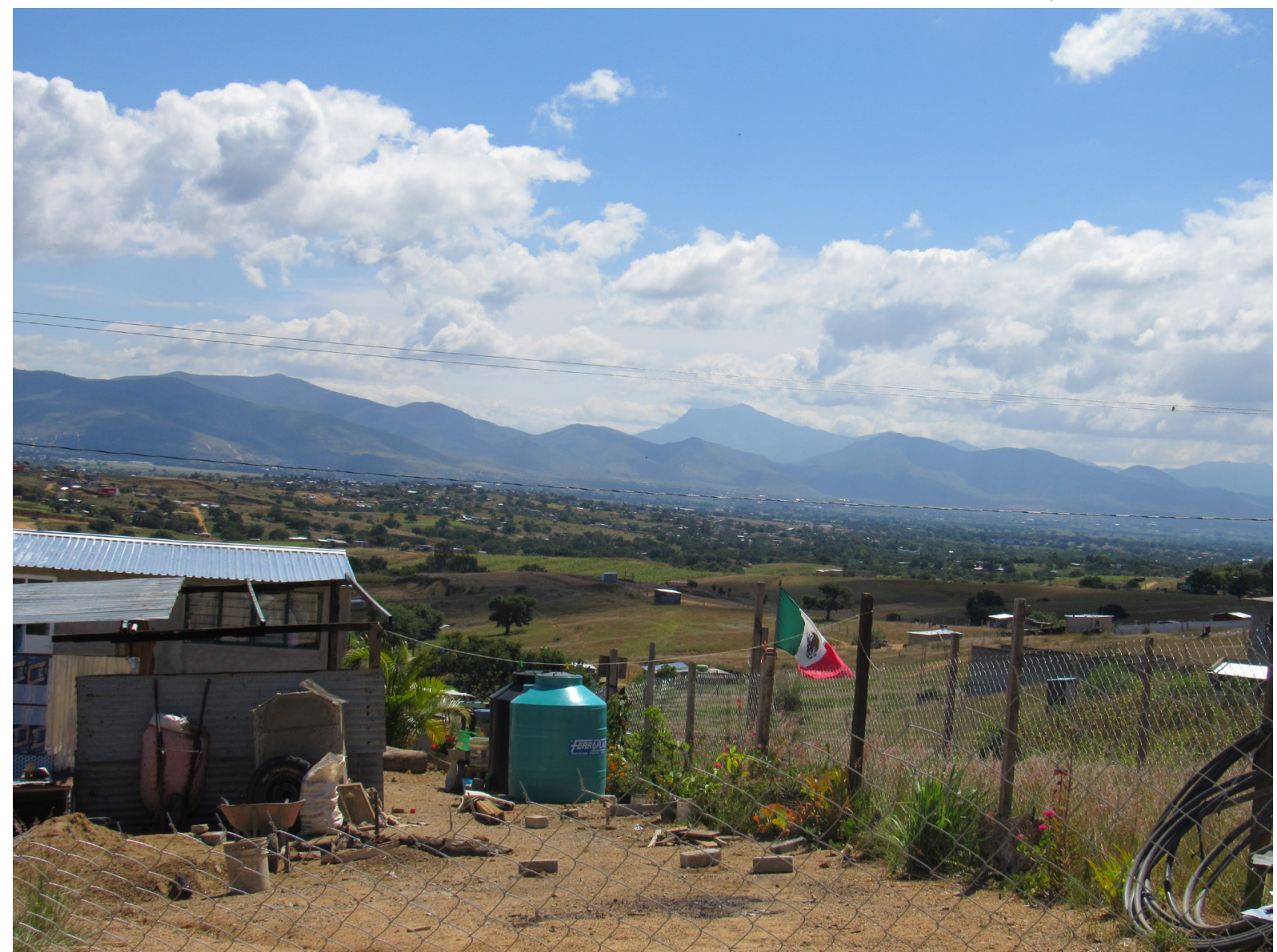

Fuente: Trabajo de campo, Oaxaca, México. 14 de octubre de 2019.

determinaron sus relaciones (horizontales, verticales y transversales) y estrategias para acceder a trabajos, instrucción escolar, oferta cultural, entre otros.

\section{CONCLUSIONES}

La aplicación de la metodología mostró resultados útiles para comprender la segregación socio-espacial en el periurbano. Sus ventajas respecto a otras metodologías permiten enfatizar las múltiples escalas (regional, municipal, localidad, barrios y familia) y temporalidades en las que se reproduce la segregación. Se pone de manifiesto que el uso de la información sociodemográfica limitada a algunos años y escala geográfica censales (AGEB, Manzana) no permite entender la complejidad del proceso, como lo mencionan otros estudios (Garrocho y Campos, 2013, p.276; Massey et.al, 2009, p.79).

La propuesta metodología también contribuye a generar conocimiento en áreas de investigación ausentes (segregación étnica y etaria) como propone
Sabatini (2006) y en el periurbano es el espacio más dinámico de las ciudades intermedias. Así mismo, el análisis de manera conjunta de la segregación física y social permitió reconocer que no son dimensiones mutuamente determinantes; una población puede estar segregada física pero no socialmente (VergaraErices y Garín, 2016). Durante la aplicación de esta metodología se han identificado dos limitantes que pueden dificultar el proceso en la recolección de datos: uno es la falta de disponibilidad de información desagregada en zonas del periurbano y, otro, el tiempo para la recolección de datos a escalas localidad, barrio y familia que es mayor debido a la necesidad de generar relaciones de confianza con los actores locales para recolectar la información. Para atender estas limitantes, esta metodología consideró otras herramientas en la recolección de información ejemplo de ellos son los mapeos participativos y las entrevistas.

Los resultados previos muestran que el acercamiento a través de múltiples escalas, temporalidades y ac- 
tores en al análisis de la segregación construye una visión compleja y más completa del proceso y el reconocimiento de las implicaciones en la desigualdad de esos espacios. La segregación es un proceso con orígenes históricos, recientemente se ha intensificado en el tiempo y en el espacio, presentándose a menores escalas y con implicaciones sociales. Los estudios mexicanos sobre segregación la visualizan como una condición física, pocos identifican su carácter multidimensional e implicaciones en la vida de la población del periurbano de ciudades intermedias.

Este documento muestra una alternativa metodológica para analizar la segregación soportada en tres pilares: primero, la multidimensionalidad que considera los aspectos físicos y sociales de la segregación, por la diferenciación de rasgos socioeconómicos, culturales, políticos y relacionales de los pobladores. Segundo, se considera que la segregación cambia en el tiempo, a través de un acercamiento multitemporal (1970, 1980, 1990, 2000 y 2010). Tercero, se retoma la multiescalaridad, reconociendo la importancia de los contextos regionales y municipales de las ciudades intermedias como ambientes en que se desenvuelven los pobladores; y centrándose en tres escalas: localidad /fraccionamiento, barrio y familia. Los resultados preliminares en dos ciudades Intermedias en México, muestra la pertinencia de esta metodología al evidenciar las particularidades e íntima relación de la segregación física y social en el periurbano. Abonando a metodologías que comprenden a la segregación como un proceso complejo, con múltiples dimensiones, escalas y temporalidades; útil para estudiar ciudades intermedias mexicanas y latinoamericanas.

\section{AGRADECIMIENTOS}

El presente estudio es parte de un proyecto denominado: la segregación en el periurbano de las ciudades medias mexicanas, los casos de Morelia y Oaxaca, investigación realizada gracias al Programa UNAMPAPIIT (IA301419).

\section{REFERENCIAS}

Aguilar, A.G. y Vázquez, M.I. (2000). Crecimiento urbano y especialización económica en México. Una caracterización regional de las funciones dominantes. Investigaciones Geográficas, 42, 87-108.

Aguilar, A. G. y Mateo, P. (2011). Diferenciación sociodemográfica del espacio urbano de la Ciudad de México. Eure, 37 (110), 5-30. doi: 10.4067/S025071612011000100001.
Aguilar, A. G., Romero, P., y Hernández, J. (2015). Segregación socio-residencial en la Ciudad de México. Dinámica del patrón territorial a nivel local 20002010. En A. Aguilar y I. Escamilla (coord.), Segregación urbana y espacios de exclusión. Ejemplos de México y América Latina (pp. 73-102). Ciudad de México, México: UNAM, Miguel Ángel Porrúa.

Álvarez de la Torre, G. (2017). Morfología y estructura urbana en las ciudades medias mexicanas. Región y sociedad, 29(68), 153-191. https://doi.org/10.22198/ rys.2017.68.a872

Aparecida, Maria. 1999. "Á GUISA DE INTRODUÇÃO." En P. Almeida y S. de Mello (coord.), Novos estudos de geografia urbana brasileira (pp.9-18). Salvador: Editorial de Universidade Federal de Bahia.

Borsdorf, A., Bähr, J. y Janoschka, M. (2002). Die Dynamik stadtstrukturellen Wandels in Lateinamerika im Modell der lateinamerikanischen Stadt. Geographica Helvetica, 57(4). 300-310. doi: https://doi.org/10.5194/gh-57-300-2002

Costa, E. y Scarlato, F. (2019). Geografía, método y singularidades revisadas en lo empírico. GEOUSP Espaço e Tempo, 23 (3), 640-661. doi: 10.11606/ issn.2179-0892.geousp.2019.161552.

Domínguez, M., (2017). Las dimensiones espaciales de segregación residencial en la ciudad de Mérida, Yucatán, a principios del siglo XXI. Península, 12 (1), 147-188.

Elorza, A. (2019). Segregación residencial y estigmatización territorial. Representaciones y prácticas de los habitantes de territorios segregados. Eure, 45(135), 91-110. doi: 10.4067/ S0250-71612019000200091.

Garrocho, C. y Campos, J. (2013). Réquiem por los indicadores no espaciales de segregación residencial. Papeles de población, 19(77), 269-300.

Garrocho, C. y Campos, J. (2015). Segregación residencial de los adultos mayores: relevancia del tema para México, marco teórico y políticas urbanas. En A. Aguilar y I. Escamilla (coord.), Segregación urbana y espacios de exclusión. Ejemplos de México y América Latina (pp. 103-138). Ciudad de México, México: UNAM, Miguel Ángel Porrúa.

Giménez, G. (1999). Territorio, cultura e identidad. Estudios sobre la Cultura Contemporánea, 5(9), 25-57.

Girola, L. (2011). Historicidad y temporalidad de los conceptos sociológicos. Sociológica, 26(73), 13-46. 
Gutiérrez, R., Díaz, K. y Román, R. (2016). El concepto de familia en México: una revisión desde la mirada antropológica y demográfica. Ciencia ergo-sum. 23(3). Recuperado de http://www.redalyc.org/articulo.oa?id=10448076002.

Hiernaux, D. y Lindón, A. (2004). La periferia: voz y sentido en los estudios urbanos, Papeles de Población, 42(10),101-123.

Hiernaux, D. (2008). De los imaginarios a las prácticas urbanas: construyendo la ciudad de mañana Iztapalapa, Revista de Ciencias Sociales y Humanidades, 64-65, 17-39.

Van Kempen, R., (2007). Divided cities in the $21^{\text {st }}$ century: challenging the importance of globalization. Journal of Housing and the Built Environment, 22(1), 13-31. https://www.jstor.org/ stable/41107366.

Madrid, G. (2011). Oaxaca, de "ciudad intermedia" a metrópoli de Los Valles Centrales. Emergencia de una ciudad-territorio en el sur de México (Tesis para obtener el grado de doctor en Urbanismo y Ordenamiento Territorial). Universidad Politécnica de Cataluña, España.

Massey, D. S., Rothwell, J. y Domina, T. (2009). The Changing Bases of the Segregation in the United States. The Annals of the American Academy, 626 (1), 74-90. https://doi. org/10.1177/0002716209343558.

Méndez-Lemus, Y., Vieyra, A., Güiza-Valverde, F. y Hernández-Guerrero, J. (2016). Relaciones sociales y expansión urbana: Aplicación del enfoque de capital social en el análisis de la adaptación de los modos de vida agropecuarios a la periurbanización. En A., Vieyra, Y. Méndez-Lemus y J. Hernández-Guerrero (coord.), Procesos urbanos, pobreza y ambiente. Implicaciones en ciudades medias y megaciudades (pp. 89-108). Ciudad de México, México: UNAM.

Pasciaroni, C. (2012). Ciudades medias: aproximación metodológica, funcionalidades y estructura productiva. Ciencias Económicas, (30)1, 399-416.

Ramírez, P. y Ziccardi, A. (2008). Pobreza, desigualdad y exclusión social en la ciudad del siglo XXI, una introducción. En R. Cordera, P. Ramírez y A. Ziccardi (coord.), Pobreza, desigualdad y exclusión social en la ciudad del siglo XXI (pp. 23-48). Ciudad de México, México: UNAM, Instituto de Investigaciones Sociales.
Roberts, B. (2015). Gestionando Sistemas de Ciudades Secundarias. Washington DC, Estados Unidos: Cities Alliance/Banco Interamericano de Desarrollo.

Rubalcava, R. y Schteingart, M. (2012). Ciudades divididas: Desigualdad y segregación social en México. México: Colegio de México.

Ruiz-López, Cinthia, Antonio Vieyra y Yadira MéndezLemus. 2021. "Segregación Espacial En Tarímbaro, Municipio Periurbano de La Zona Metropolitana de Morelia, Michoacán, México." Revista de Geografía Norte Grande (78):237-57. doi: 10.4067/ S0718-34022021000100237.

Sabatini, F. (2006). La segregación social del espacio en las ciudades de América Latina. Recuperado de https://publications.iadb.org/es/la-segregacionsocial-del-espacio-en-las-ciudades-de-americalatina.

Saraví, G. (2004). Segregación urbana y espacio público: los jóvenes en enclaves de pobreza estructural. Revista de la Cepal, 83, 33-48. doi: 10.18356/ f9966207-es.

Sartre, J. (1960). Crítica de la razón dialéctica. Buenos Aires: Editorial Losada.

Savage, M., Devine, F., Cunningham, N., Taylor, M., Li, Y., Hjellbrekke, J., Le Roux, B., Friedman, S. y Miles, A. (2013). A New Model of Social Class? Findings from the BB'C Great British Class Survey Experiment. Sociology 47(2):219-50. doi: 10.1177/0038038513481128.

Scarlato, F. y Costa, E. (2017). A Natureza Do UrbanoLa Nature de l'urbainThe Nature of Urban. Confins (30). doi: 10.4000/confins.11676.

SEDESOL (Secretaría de Desarrollo Social) y ONU-HABITAT. (2011). Estado de las ciudades de México. Rio de Janeiro. México: SEDESOL, ONU-HABITAT.

SEDESOL (Secretaría de Desarrollo Social), CONAPO (Consejo Nacional de Población) e Instituto Nacional de Estadista y Geografía (INEGI). 2012. Catálogo sistema urbano nacional 2012. México D.F.: CONAPO. http://www.conapo.gob. $\mathrm{mx} / \mathrm{en} / \mathrm{CONAPO} /$ Catalogo_Sistema_Urbano_ Nacional_2012

SEDATU (Secretaría de Desarrollo Agrario, Territorial y Urbano) y CONAPO (Consejo Nacional de Población). 2018. Sistema Urbano Nacional 2018. México D.F.: SEDATU / CONAPO. Recuperado de https://www.gob.mx/conapo/documentos/sistema-urbano-nacional-2018. 
Thibert, J. y Osorio, G. (2014). Urban Segregation and Metropolitics in Latin America: The Case of Bogotá, Colombia. International Journal of Urban Regional Research, 38.4, 1319-1343. doi:10.1111/1468-2427.12021.

UCLG (United Cities and Local Governments) y (CGLU) Cité et Gouvernements Locaux Unis. (2016). CoCreando el Futuro Urbano. La Agenda de las Metrópolis, las Ciudades Intermedias y los Territorios. GOLD IV. Recuperado de https://www.uclg.org/

\section{NOTAS AL FINAL}

1 Algunos consideran que estas urbes van de 50,000 a $1 \mathrm{mi}-$ llón de habitantes y otros, más de 500 mil habitantes (Roberts, 2015). En América Latina y el Caribe las ciudades de mediano tamaño están principalmente en el rango de 300 mil a 1 millón (UCLG y CGLU, 2016).

2 Algunos avances del proyecto están en proceso de publicación en un capítulo de libro: Propuesta teórico-metodológica para analizar la segregación en el periurbano de las ciudades medias mexicanas. En Expansión del periurbano y conformación de territorios desiguales.

3 La mayoría de los análisis utilizan las Áreas Geoestadísticas Básicas para revisar la segregación (el nivel más básico de desegregación de la información estadística y cartográfica en las ciudades mexicanas), pero no indagan sobre las múltiples relaciones (sociales, culturales, políticas, ambientales, económicas) en su interior.

$4 \quad$ El autor no estudia el periurbano ni la segregación, pero sus planteamientos filosóficos sobre el sujeto son útiles para soportar esta propuesta metodológica. sites/default/files/gold_iv_resumen_ejecutivo. pdf.

Vaughan, L. y Arbaci, S. (2011). The Challenges of Understanding Urban Segregation. Built Environment, 37 (2), 128: 138. doi: 10.2148/ benv.37.2.128.

Vergara-Erices, L. y Garín, A. (2016). Vivienda social y segregación socioespacial en una ciudad pequeña: el caso de Angol, Chile. Polis, No 44, 1-25. doi: $10.4067 / \mathrm{s} 0718-65682016000200021$.

5 Otro término, ha sido el suburbano, ampliamente usado en México hasta la década de los setenta del siglo XX, para referirse a los espacios residenciales de la periferia de clases altas (Hiernaux y Lindón, 2004, p.103; Hiernaux, 2008, p.21). El suburbano ve a lo rural como espacio vacío sobre el cual se expande la ciudad, (Borsdorf, Bähr y Janoschka, 2002).

$6 \quad$ En México, a partir de 1990 se tiene disponible la información sociodemográfica desagregada por localidades.

7 Las escalas de los patrones de segregación en el barrio se construirán con la información disponible por municipio periurbano, es importante aclarar que en México la disparidad en la información entre periurbano y ciudad consolidad es una constante.

8 En México, la información sociodemográfica a nivel de manzana está disponible solo hasta el 2010.

9 Más información sobre los resultados se encuentra publicados en (Ruiz-López, Vieyra y Méndez-Lemus, 2021). 